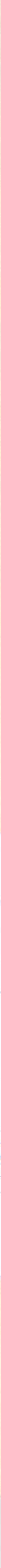




\title{
DIDÁCTICA DE LA ESCRITURA CREATIVA
}

\section{Didactics of creative writing}

\author{
Raxson Montilla Narváez ${ }^{1}$ \\ 1. E. Gimnasio Gran Colombiano \\ Email: ${ }^{1}$ raxson.montilla@uptc.edu.co
}

(Recibido el 24 de mayo de 2021 - Aceptado el 08 junio de 2021)

\section{Resumen}

Este artículo tiene por objeto definir una didáctica de la escritura creativa basada en la conceptualización epistémica de la didáctica general y la delimitación de su objeto de estudio. Entendiendo, que enseñar escritura creativa exige al docente convertirse en un agente de contagio, conocer la literatura, los procesos de producción escrita y las reglas formales de la lengua. Además, como proceso creativo, permite el desarrollo del pensamiento del estudiante, sujeto activo y autónomo que construye y aporta a su aprendizaje, uno que trascienda las fórmulas de producción, incentivando el desarrollo de una voz propia y representando el territorio.

Palabras clave (Tesauro de la Unesco): educación, escritura creativa, técnica didáctica, situación del docente, enseñanza, formación.

\begin{abstract}
This article aims to define a didactics of creative writing based on the epistemic conceptualization of general didactics and the delimitation of its object of study. Understanding that teaching creative writing requires the educator to become an active agent, to know the literature, the written production processes and the formal rules of the language. Furthermore, as a creative process, it allows the development of the student's thinking, an active and autonomous subject that builds and contributes to their learning, one that transcends the formulas of production, encouraging the development of their own voice and representing the territory.
\end{abstract}

Keywords (Unesco thesaurus): education, creative writing, classroom techniques, teacher status, teaching, training.

Entender el papel de la didáctica dentro del proceso educativo ha sido un desafío que han afrontado grandes teóricos a lo largo de la historia. Esto significa que la categoría 
didáctica ha sufrido transformaciones debido a su naturaleza epistémica (Castaño y Fonseca, 2008). ¿Es una ciencia con todo lo que eso implica? ¿Es una disciplina científica con su propio objeto de estudio y escenario investigativo? ¿Es simplemente un adjetivo que da nombre a un hacer práctico? ¿Es un campo del saber? Preguntas fundamentales al momento de pensar la didáctica y su objeto de estudio.

Del mismo modo, pensando en la complejidad de la didáctica, es necesario analizar algunos conceptos como el triángulo didáctico que propone Astolfi (2001), su relación con la transposición didáctica de Chevallard (1997) y las dimensiones del conocimiento de las que hablan Grossman, Wilson y Shulman (2005). Teorías que sirvieron como punto de partida para la construcción de esta propuesta didáctica, la cual, teniendo en cuenta que trata específicamente la escritura creativa, entabla relaciones con posturas de autores como Cassany (2016) y su propuesta de escritura extensiva; la didáctica de la escritura creativa de Alonso (2001); la teoría literaria como herramienta de enseñanza (Bordons y Díaz-Plaja, 2006); el contagio literario que plantea Altamirano (2016); la experiencia de la sed de exploración de la Maestría en escritura creativa del Instituto Caro y Cuervo (Álvarez y Cárdenas, 2019); y la idea de fomentar la escritura creativa desde la educación primaria de Arroyo (2015).

\section{LA NATURALEZA EPISTÉMICA DE LA DIDÁCTICA}

Comenio, en el siglo XVII, parece ser el primero en acuñar el término, desde el cual planteó algunas preguntas: ¿Qué enseñó? ¿Para qué enseñó? ¿Cómo enseñó? ¿Con qué enseñó? Se presenta, entonces, la primera preocupación de la didáctica: la enseñanza. No obstante, debido a su momento en la historia y su lejanía con la educación actual, su visión del acto de enseñar es un poco limitada. Una visión más reciente es la de Zambrano (2005), que la ve como disciplina científica, idea francófona que habla de las ciencias de la educación, poniéndola a la altura de la filosofía, la antropología y la sociología, por nombrar algunas. Esto genera algunas responsabilidades: definición de un campo específico, necesidad de un desarrollo de teorías e instrumentos investigativos propios, independencia respecto a la pedagogía.

Por otro lado, se encuentra el punto de vista de Runge (2013), que la define como subcampo de la pedagogía, algo que parece reafirmar Vasco (1990), quien plantea que la didáctica hace parte del saber pedagógico, precisamente de lo que se refiere a la enseñanza (Castaño y Fonseca, 2008). Camilloni (2007, p. 22), también manifiesta que "la didáctica es una disciplina teórica que se ocupa de estudiar la acción pedagógica", la diferencia con los autores anteriores es que se preocupa por los problemas esenciales de la educación como práctica social y no solo por la enseñanza. Aquí toma importancia Astolfi (1998), que propone ver la didáctica como un sustantivo y no como adjetivo, ya 
que no se trata de algo sistemático, lineal, progresivo sino de un campo de estudio que analiza un fenómeno social de enseñanza y aprendizaje.

Sin embargo, una postura que genera tensión entre las ideas epistemológicas sobre la didáctica y que la alejan de la idea de ciencia es la que propone Zuluaga (2003, p. 32), quien afirma que esta "es un conjunto de conocimientos, referentes a enseñar y a aprender que conforman un saber". Por consiguiente, podría decirse, entonces, que "la pedagogía, lo mismo que la didáctica, en cuanto saberes implícitos en la práctica de los maestros, son más bien objeto de reflexión arqueológica y genealógica, y que también, pueden considerarse como disciplinas de segundo orden" (Vasco, Martínez y Vasco, 2008, p. 34). Esto significa que la didáctica todavía necesita una epistemología sólida "no para ser juzgante, determinante, definitorio, sino para buscar su génesis, sus hilos, sus redes y su constitución actual, en la cual se ha construido una positividad: conceptos y nociones, métodos y técnicas" (Vasco, Martínez y Vasco, 2008, p. 36).

\section{EL OBJETO DE ESTUDIO DE LA DIDÁCTICA}

Aunque en un comienzo Comenio se centró en la enseñanza, Meyer según Runge (2013), fue más allá y agregó dos dimensiones: la interacción entre los sujetos y los conocimientos y el aprendizaje, ya que dentro de la práctica educativa no solo se enseña, también se lleva a cabo una interacción que permite el desarrollo de conocimientos, habilidades y destrezas. Para explicarlo, Runge se basa en la teoría reconstructiva de la didáctica Flechsing (1983), que propone tres niveles del proceso didáctico: 1. Como práctica, se centra en la observación del quehacer del acto de la enseñanza; 2. Como teorización, reflexiona sobre esa praxis; 3. Relación y reflexión de las teorías pertinentes para el desarrollo de la enseñanza, respondiendo a las preguntas del cómo podría o debería ser. Del mismo modo, Castaño y Fonseca (2008), afirman que la didáctica "se pregunta por acerca de cómo conoce el hombre y de cómo el maestro configura procesos de proximidad a los diversos saberes institucionalizados o no" (p. 85). Una definición que agrega, al objeto de estudio, algunas reflexiones, por ejemplo, Zambrano (2006), define cuatro: la comprensión de la ciencia, los conocimientos científicos que se desplazan hacia la escuela para ser enseñados, una gramática para pesar las condiciones sociales y políticas del saber científico, su recorrido dentro de la escuela y el reconocimiento de lo específico de cada saber.

\section{CONCEPTOS SOBRE LA DIDÁCTICA Y SUS RELACIONES}

Situación didáctica. Zambrano (2005), se refiere a esta categoría como al conjunto de condiciones que tienen lugar en el aula de clase, cuyo rol es determinante en los procesos de aprendizaje y en la adaptación de los contenidos. Por ende, se empieza a pensar qué 
pasa con la relación de los sujetos con el conocimiento y las condiciones que rodean la construcción de conocimiento por parte del estudiante.

La reflexión didáctica. Cabe aclarar que este concepto no se atribuye a ningún autor en particular, pero sí es necesario pensando en algunas preguntas que plantea la didáctica: ¿Qué relaciones se establece entre los sujetos maestros y estudiantes? ¿Qué relaciones se dan entre los sujetos y los objetos del saber? ¿Qué relaciones se producen entre el conocimiento erudito, el conocimiento enseñable y los conocimientos particulares? Por ejemplo, Astolfi (1998), señala que comprender esas relaciones pasa por una reflexión psicológica, social, pedagógica y epistemológica. Desde su postura del triángulo didáctico propone las relaciones entre tres vértices que ocupan los actores del proceso educativo: enseñante, alumnos y saberes.

\section{Figura 1.}

Esta imagen se basó en el triángulo didáctico de Astolfi, pero se realizaron modificaciones interpretativas.

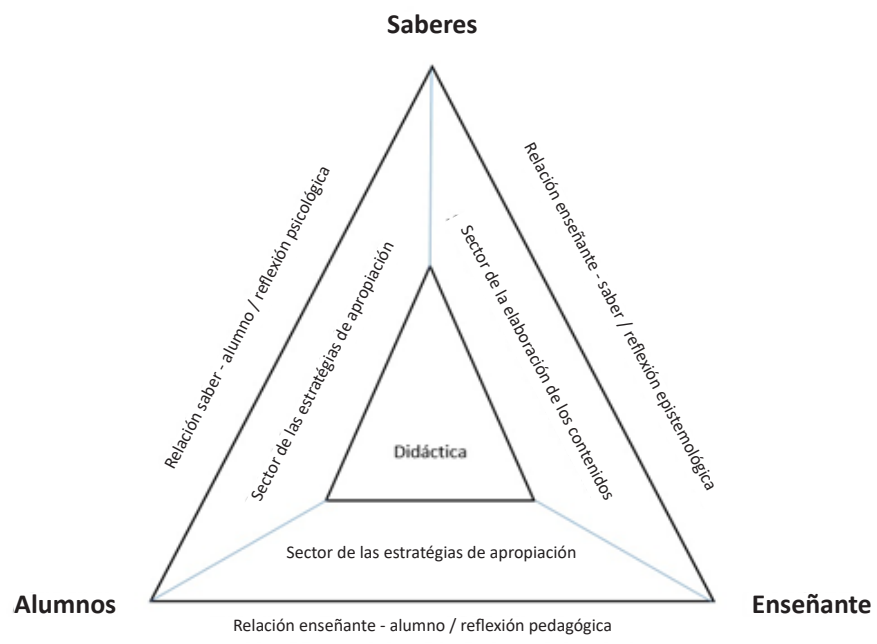

Source. Autores

Como podemos ver en la Figura 1, en el vínculo entre enseñante y alumno se presenta las interacciones didácticas y se hace la reflexión pedagógica, la cual va encaminada a pensar en la situación problema, la transmisión, el contrato didáctico y la estructuración. La relación alumno-saber requiere una reflexión psicológica y social, preguntando ¿cómo el alumno adquiere ese saber? ¿Cómo se apropia del mismo? ¿Cuáles son las representaciones que el alumno tiene del conocimiento a aprender? Por último, la relación del enseñante con el saber, el cual se entiende como una construcción que tiene un sentido histórico, cultural, 
social y por supuesto epistemológico, por tanto, es necesario preguntarse: ¿cuál y por qué ese saber? ¿cuál es la naturaleza epistémica de ese saber?

A partir de estas relaciones, surge un concepto que, aunque retoma Astolfi (2001), es una propuesta de Chevallard (1997), la cual señala que hay un saber sabio o científico que debe ser enseñado, entonces cuando pasa al ámbito de lo escolar sufre lo que se denomina una transposición didáctica. Esta transformación se da porque la intencionalidad es distinta en cada proceso. No podemos pensar que los ritmos, los objetivos, los intereses sean los mismos; se debe entender que la relación maestro alumno, maestro conocimiento y alumno conocimiento no puede ser la misma que la del científico con su práctica. Se construye el saber escolar.

Como podemos ver, "la enseñanza implica una traslación del conocimiento de la materia per se en conocimiento materia para la enseñanza" (Grossman, Wilson y Shulman, 2005, p. 7). Sin embargo, estos autores van más allá de la teoría de Chevallard y definen unas dimensiones del conocimiento. Toman en consideración el concepto de McEwan (1987) de interpretación didáctica de la materia, que tiene que ver con el pensamiento didáctico de Feiman- Nemser y Buchmann (1985) y proponen reconocer el conocimiento de sí mismo (enseñante), de los niños (alumnos) y de la materia (saberes).

Lo que sugieren Grossman, Wilson y Shulman (2005), es que se debe entender que cualquier tipo de conocimiento de la materia afecta tanto el proceso de instrucción (el cómo), el contenido mismo (el qué) y el tipo de aprendizaje que alcanza el estudiante. Al fin de cuentas, la interpretación de la materia que haga el enseñante puede convertirse en una instrucción que genere una réplica y no una verdadera construcción de conocimiento que se pueda aplicar al contexto estudiantil. Por tal razón, definen las dimensiones del conocimiento:

El conocimiento del contenido. Es la información objetiva, la organización de principios y de conceptos centrales de la materia. Permite que tanto el maestro como los estudiantes construyan puentes entre contenidos de la misma o entre diferentes materias. Cabe resaltar que el alto o bajo conocimiento del contenido afecta la instrucción. Aunque no se puede saber todo, "los futuros profesores deben entender la centralidad del conocimiento del contenido para la enseñanza y las consecuencias de una falta de conocimiento" ( $p$. 13).

Conocimiento sustantivo de la enseñanza. Es la estructuración de la interpretación de la materia, ¿cómo guiar la indagación y pensar en la discusión de cada disciplina? Este tipo 
de conocimiento impacta directamente la acción didáctica, ya que trata de dar sentido a los datos que ofrece el estudio de una disciplina.

Conocimiento sintáctico de la enseñanza. Esta dimensión introduce el conocimiento en el contexto, se dan las bases para entender los medios que le permiten a un saber ser introducido y aceptado en una comunidad puntual.

Creencias acerca de la materia. Muchas veces las creencias son tomadas como conocimiento, lo que afecta la enseñanza. Las creencias se basan en la afectividad y la subjetividad e influyen en lo que se elige enseñar y en el cómo se lleva a cabo. Muchas veces estas llevan a definir lo que es importante y a dejar al lado partes fundamentales del conocimiento del contenido.

En conclusión, el desafío es la transformación de un conocimiento disciplinar a una forma de conocimiento apropiada y específica para la tarea de enseñar, integrando la acción didáctica, la transposición didáctica, las reflexiones sociales, psicológicas, pedagógicas, epistemológicas y las dimensiones del contenido en la práctica docente.

\section{DIDÁCTICA DE LA ESCRITURA CREATIVA}

Para empezar, de acuerdo con Alfonso (2008), la didáctica de la escritura creativa es un escenario poco estudiado, existe "un bachillerato o una licenciatura encaminada a las artes plásticas, música, cine, danza, teatro... pero no se reconoce el aprendizaje de la escritura literaria y en consecuencia tampoco se reconoce su enseñanza" (p. 51). Quizá, porque "crear un texto literario es un acto complejo tanto para aquellos escritores que se dedican a la escritura de manera profesional como para cualquier otra persona que se halle frente a un papel en blanco" (Arroyo, 2015, p. 6). Sin embargo, como propone Zambrano (2005), es necesario que se aborde el estudio y la investigación de este saber específico.

Pero, ¿qué es la escritura creativa? Por un lado, está la escritura, según Ong (2006), "sistema codificado de signos visibles por medio del cual un escritor podía determinar las palabras exactas que el lector generaría a partir del texto" (p. 86), una tecnología que se puede aprender a usar, un proceso consciente que posibilita una mirada reflexiva a través de la distancia que hay entre el escritor y el texto. Por el otro, la creatividad, uno de los tres aspectos esenciales de la inteligencia exitosa (Sternberg, 1997) (los otros dos son el analítico y el práctico), definida por Velásquez, Remolina de Cleves y Calle (2010, p, 326) como: conjunto de capacidades intelectuales, afectivas y motoras propias del individuo que a través del proceso educativo se pueden manifestar mediante 
la estimulación, motivación intrínseca y extrínseca para aportar soluciones originales a problemas determinados, así como crear con ingenio y transformar a partir de lo que ya se tiene.

Entonces, se podría afirmar que la escritura creativa tiene mucho de lo que dice TimbalDuclaux (1993, p. 11): "escribir en el más noble sentido del término: de componer, de crear. Y el problema de fondo es el de inspiración; el punto en el que el arte de escribir toca íntimamente el arte de pensar", porque depende, como proceso individual, de "las capacidades cognitivas, relacionales, emocionales, afectivas, valorativas, construidas por lo humano en su largo devenir" (Gorz citado por Mejía, 2020, p. 117), y como ejercicio subjetivo, del reconocimiento de las habilidades específicas como creatividad, intuición, reflexión, pensamiento lógico, sensibilidad y observación. No obstante, como ejercicio de producción textual, requiere el desarrollo de algunas competencias: lingüística, sociolingüística o de adecuación, discursiva y estratégica (Canales y Swain, 1981). Como lo relatan Álvarez y Cárdenas (2019, p, 124):

Pensar hoy la escritura creativa quizá sea clausurar, por ejemplo, la metáfora de la carpintería, que tiene la gracia de haber marcado una época y la desgracia de habernos hecho creer, en un momento en el que quizá eso era lo que necesitábamos creer, que la escritura es un asunto manual, cuando escribir, por más técnicas que se identifiquen y se distingan -en pro de la claridad pedagógica o de la venta de manuales para hacerlo rápido-, no deja de ser, sustancialmente, una actividad intelectual.

De tal modo, es necesario pensar en un planteamiento epistemológico fundamentado, no solo en una teoría desde la escritura creativa sino desde la didáctica y sus relaciones.

\section{Reflexiones didácticas}

¿Por qué ese saber debe ser enseñado? De acuerdo a Giraldo (2015, p. 42), la escritura "además de servir de puente para conocer o dar a conocer, también reforma la conciencia; es decir, que la expresión escrita tiene efectos en los pensamientos y sentimientos de quien la emite". Agrega también, que es una forma de construcción del mundo y una forma de existir, porque la escritura tiene múltiples razones: "intelectual, social, cultural a lo estético y lo moral" (p. 43).

Respecto a la escritura creativa, según Alonso (2008), promueve el crecimiento psicológico e intelectual y permite una relación social, ya que contrasta el imaginario con la realidad. Arroyo (2016), por su parte, habla del fortalecimiento de las funciones de la escritura que propone Cassany (1999): funciones intrapersonales, las cuales van encaminadas al ejercicio de escribir para sí mismo, por ejemplo: para recordar, manipular información, 
crear ideas o ampliar conocimientos; las funciones interpersonales: escribir para otros sea para interactuar, contar relatos u organizar la sociedad; y la función estética o lúdica, que puede ser intrapersonal e interpersonal, no importa si es dirigido a otro o no, solo busca el deleite. Como afirman Bordons y Díaz-Plaja (2005, p. 13):

Sea con un trabajo analítico previo, sea a partir del texto o sea tomado como punto de partida una brainstorming o un diálogo entre el alumnado, la escritura creativa "enseña» a menudo más que grandes lecciones magistrales sobre el proceso que han seguido los grandes escritores para construir sus obras.

Por esta razón, la enseñanza de la escritura creativa aporta a la educación literaria, estética, intelectual, subjetiva, intra e inter personal. Esto permite el desarrollo de capacidades y habilidades que aportan al perfeccionamiento de las competencias comunicativas y textuales.

Por ejemplo, Díaz (2016), a través del análisis de experiencias con niños entre los 6 y los 9 años, asegura que la práctica de la escritura creativa se fundamenta en cuatro aspectos: participación activa de los padres; la escritura percibida de otra manera: "interés por expresar las experiencias personales, recuerdos, sentimientos, ideas y emociones" ( $p$. 67); ayuda a afianzar la autonomía, la autoestima y el autoreconocimiento promoviendo el pensamiento y la acción crítica (p. 70); incursionar en el mundo de la imaginación, la fantasía y la creación, renovando prácticas habituales de escritura en la escuela (p. 71).

Otra propuesta, pensando en la "creatividad (como todo proceso psicológico)" que "debe ser abordada longitudinalmente, es decir, como una función en despliegue permanente" (Alessandroni citando a Vygotski, 2017, p. 56), es la de Cassany (2016):

Las actividades intensivas se plantean principalmente objetivos de tipo actitudinal (fomentar hábitos, cambiar actitudes, desarrollar motivación, etc.) a partir de la utilización de textos extensos durante un periodo dilatado de tiempo. Suelen dar más protagonismo al aprendiz, aunque sea el profesor el que proponga y organice la actividad. También pueden integrar contenidos de materias no lingüísticas (p. 94).

Como lo afirma Delmiro Coto (2002, p. 17), la escritura creativa y literaria no es algo inútil porque aporta al desarrollo del estudiante, no solo desde la visión formal de la escritura sino desde el desarrollo del pensamiento y las emociones. La fantasía y la imaginación se vuelven una salida al peso de lo cotidiano; el fracaso es una herramienta formadora frente a la mera existencia y a la desgracia; la construcción del ser físico, emocional y social genera una valoración crítica del ser y del mundo; además, muchas veces ayuda a 
encontrar sentido a la vida y a comprender el mundo; ayuda al manejo de la información en un escenario inundado por los medios de comunicación; fomenta el conocimiento de la cultura y de la literatura.

Por otra parte, integrando a la discusión la relación del enseñante con el aprendiz, Alonso (2008), propone que desde el comienzo se entable un diálogo para definir los objetivos. Plantea, además, que entre los participantes se conteste a las preguntas cómo se escribe, quién escribe, para qué se escribe, desde dónde se escribe, a quién se escribe, cómo se aprende y quién enseña. "La enseñanza de la escritura ha de ser personalizada otorgando a los alumnos la posibilidad de escribir sus preocupaciones, ideas, recuerdos y sentimientos" (Arroyo, 2016, p. 24). Altamirano (2016, p. 168-169), desde otro punto de vista, habla de "un sentimiento estético verbal del profesor de literatura hacia los alumnos, a través del acto de la comunicación literaria modélica, para despertar en ellos el entusiasmo literario que conduzca al disfrute de la literatura".

De acuerdo a Cassany (2016), el profesor no dirige al alumno, este es el protagonista o centro. No se trata de apuntar a un producto sino al proceso de composición, se debe relacionar la escritura con los temas del currículum y con la experiencia. Fomentar la colaboración entre los actores del proceso formativo. Al igual que la postura anterior ese debe favorece la autonomía y la responsabilidad del alumno, el proceso debe ser flexible y plantear una coevaluación formativa.

Por consiguiente, se debe propiciar una relación lúdica, experimental y estética con el lenguaje que desbloquea el imaginario, fomentando habilidades creadoras propias y de la estética de lectura y escritura; aunque es un proceso autónomo el que activan la creación, puede implicar un trabajo grupal; el docente invita a observar la realidad desde una perspectiva poética, fomenta la oralidad, genera procesos de escritura y otorga gran valor a la lectura. Es una alternativa al uso del lenguaje en la escuela (Álvarez, 2007, p. 84-85). Como propone Briz (2003, p. 296): "ha de procurar un objetivo más ambicioso todavía: hacer posible que el aprendiz busque su propia voz, su propia expresión, para que la construcción de un discurso personal sea una perspectiva, primero, deseada y, después, sentida como necesidad".

\section{Situación didáctica}

Respecto a la relación de los sujetos con el conocimiento, Altamirano (2016), usa el ejemplo del virus donde el enseñante contagia a sus aprendices el goce de la literatura y los estudiantes, como sujetos activos, construyen y aportan a su aprendizaje. Este autor, citando a Not, "propone la educación en segunda persona ("interestructuración"), fundada en la dialéctica hegeliana y en el interaccionismo social de Vygotski"(p. 159). 
Por esta razón, es claro que la relación del estudiante con la escritura creativa y la pasión por escribir literariamente se puede infectar, pero también se construye a través de las experiencias de los sujetos con sus territorios y con otros individuos. Peña y Quintero (2015, p. 204), afirman que esto "implica al docente brindar espacios de escritura auténtica para lograr que los niños sean sujetos activos de la comunidad escrita a la cual pertenecen y conscientes de su propio proceso escritor".

Respecto a las condiciones que rodean la construcción de conocimiento, Alonso (2008, p. 58), manifiesta que se debe "buscar o crear un espacio didáctico específico desde donde plantear desde otra perspectiva las relaciones entre ciencias del lenguaje y la literatura para hacer una didáctica que trabaje lo imaginario y la creatividad". Como dice Rosales (2010, p. 2) se debe partir de "la concepción del aula como un espacio de comunicación, un canal adecuado para el desarrollo de las competencias comunicativas. En este sentido nos parece importante la planificación y el desarrollo de propuestas de intervención didáctica que fomenten dichas competencias". Planteamiento que encamina la discusión hacia la construcción de nuevos escenarios educativos, que permitan mejores condiciones para pensar no solo la enseñanza de la escritura creativa sino de la enseñanza en general. Mejía (2020), por su parte, se refiere a estas condiciones a través de "las capacidades como fundamento y eslabón clave para el desarrollo de las habilidades culturales que soportan los talentos con los cuales nos movemos en la vida, y ese saber hacer propio que se da en los contextos" (p. 46). De este modo, las condiciones están dadas por el fortalecimiento de las capacidades en los entornos particulares, las experiencias de los sujetos y sus habilidades y las relaciones que plantean los territorios, los mismos individuos y el conocimiento.

\section{Transposición didáctica}

Altamirano (2016, pp. 168-169), afirma que "el sentimiento estético literario no se puede enseñar; solamente se puede transmitir o contagiar porque la experiencia literaria no puede reproducirse mediante operaciones puramente cognitivas". Por ende, debe pensarse desde lo estético, "consiste en adoptar una actitud emotiva, no racional, que pone en juego lo sensorial, lo afectivo, lo cualitativo: los sentidos, los sentimientos, las emociones y las intuiciones". Esto significa que la relación del enseñante con el conocimiento se hace a través de una mirada subjetiva; la cual permite, por medio de la percepción estética y la interacción con el aprendiz, su transmisión. El deleite que expresa el docente sirve como estímulo y contagio para el estudiante. Un proceso que transforma del pensamiento y del actuar literario. Por esta razón, de acuerdo a Alonso (2008), esta transposición estaría encaminada a la interpretación del estudio de la literatura, no solo de su lectura sino de la escritura de textos de intención literaria. 
Respecto al contenido y a la metodología se debe tener en cuenta: "la lingüística textual aplicada a la enseñanza de lenguas, la teoría de la literatura, la psicología del aprendizaje y planteamientos desde las didácticas específicas" (Alonso, 2008, p. 56). Permitiendo, de este modo, la relación epistémica con el saber, idea de Astolfi (1998) y de Zambrano (2005).

De acuerdo con Álvarez y Cárdenas (2019), esta relación con el conocimiento genera tensión entre la escritura creativa, la literatura y el mismo lenguaje. Por ende, una pregunta que plantea su experiencia es "¿de qué modo, por ejemplo, el lenguaje, la literatura, podían ser el acta conjetural de un territorio?" (p. 127), una mirada social y política de la escritura creativa, de la que surgen las siguientes ideas: "el territorio es eso que no se puede reducir a una mercancía, como el lenguaje", "escribir es volver a recorrer el territorio" (p. 129). Así, el texto se ve como un territorio que reconstruye la historia, que se vive y revive cada vez que se lee.

Tavarovsky (2010), por su parte, asegura que "la escritura y el libro se oponen porque sobre ellos operan poderes diferentes. Sobre la escritura influyen la indiferencia, el cansancio, el exceso. Sobre el libro, la tapa, el comentario, la circulación" (p. 99). Una idea bastante controversial que aleja el ejercicio solitario de la escritura de la obra en sí misma, ya que el primero es la experiencia de la creación como instante que acontece ahora y la segunda la materialización, la suspensión del mundo (pp. 139-140).

En consecuencia, la relación docente/saber debe hacerse desde una mirada crítica de la escritura creativa y una vocación política, que permita "acercarse a aquellos escenarios sociales donde están en negociación los términos de constitución del mundo de una cultura y una comunidad, y atravesarlos con la imaginación; atravesarlos y remozarlos y expandirlos desde las voces propias de los sujetos (Álvarez y Cárdenas, 2019, pp. 123124)".

\section{Dimensiones del conocimiento}

Citando a Cassany (2016) y pensando en el conocimiento del contenido, los contenidos estarían encaminados a las estrategias de composición de textos donde "la necesidad es formar los aprendices-autores en habilidades cognitivas específicas de escritura (buscar información, saber estructurarla, saber adaptarla al lector, etc.)” (p. 102). Como lo afirma Corrales (2001, p. 75), el docente debe "conocer e investigar a fondo los fundamentos de la creación literaria":

la diferencia entre expresión, información y opinión como partes integrantes de un texto; las distintas funciones que cumple el lenguaje en el interior de un discurso; el 
conocimiento y reconocimiento de las zonas expresivas a fin de localizar la percepción y ordenar el pensamiento creativo; el trabajo sobre diferentes áreas de la escritura: relación entre conciencia y sentidos, la representación, los diferentes lenguajes y modos de la descripción, los aspectos que conforman la teoría del punto de vista, la construcción de la voz narrativa, el dialogismo y los sistemas de acotaciones, el monólogo interior, los flujos de conciencia, la creación de personajes, tramas y argumentos, etc.

Pero este autor no considera solo estas cuestiones, agrega: los docentes deben contar con unos criterios mínimos que permitan guiar y estimular a los alumnos en su trabajo creativo; ser creativos a la hora de la práctica, planteando ejercicios colectivos e individuales donde se integren diferentes modos de expresión; tener un amplio conocimiento de la literatura que permita añadir la lectura como eje fundamental de la escritura; tener "buena formación en técnicas de corrección y anotación de ejercicios escritos" (p. 76), que permita interpretar, corregir y evaluar de manera coherente los ejercicios escritos de los estudiantes, respetando las particularidades que ofrecen cada texto desde una mirada no solo lingüística o discursiva sino como obra artística.

Entonces, el enseñante debe pensar las tareas de escritura de acuerdo a las capacidades de los alumnos, pero sobre todo buscar herramientas que fomenten el autoaprendizaje y la práctica escritural. Por tanto, el contenido escritura creativa estaría centrado en la literatura y su producción; pero no solo desde la mirada histórica de la literatura y formal de la escritura, sino desde una mirada subjetiva y estética. Por ejemplo, Alonso (2008), hace una crítica al saber que se transmite normalmente en la enseñanza de la literatura, donde se plantea una visión descriptiva que enseña épocas literarias, autores y obras, dejando al lado "los conocimientos literarios previos de los alumnos y de la situación estética en que se produce la lectura del texto" (p. 58). Así pues, parece que no se tiene en cuenta el conocimiento sustantivo a la hora de pensar en qué es lo importante de la literatura en cuanto herramienta para la enseñanza de la escritura creativa.

Respecto al conocimiento sintáctico de la enseñanza, Arroyo (2016) y Álvarez y Cárdenas (2019) hablan de "contagiar" a los alumnos. El primero hacia el interés por el entorno, formando escritores críticos e inquietos respecto a lo que los rodea; los segundos, hacia la pasión por la lectura y la escritura literaria. Así mismo, para Cassany (2016), es mejor que sea una historia real y cercana al alumno, no solo verosímil, ya que esto permite una relación directa con el contexto, "es importante que las situaciones propuestas coincidan con las necesidades y los intereses del aprendiz (temas, propósitos comunicativos, etc.)" (p. 100).

Adicionalmente, aunque es importante que el enseñante transmita la pasión a sus aprendices, esto no significa que pueda dejar de lado conocimientos por simples creencias acerca de la materia, no se pueden confundir con el placer por la escritura y la literatura. 
Un ejemplo claro de esta idea, es el "giro auditivo", que a través de una "indagación en las ontologías y epistemologías de lo acústico" realizada por Ana María Ochoa, esta se dio cuenta que se han dejado por fuera muchos conocimientos por no pertenecer a la tradición letrada: "pronunciamientos salidos de tono, difíciles de clasificar como lenguaje o como canción; acentos impropios del español; sonidos de lenguas indígenas sin signos equivalentes en el alfabeto español; o una abundancia de ruidos y voces provenientes de entidades naturales que sobrecargaban los sentidos" (Álvarez y Cárdenas, 2019, p. 124).

\section{CONCLUSIÓN}

El campo de la enseñanza y el aprendizaje de la escritura creativa está cubierto por un manto de divinidad artística que hace que una propuesta de didáctica parezca una utopía. Por tanto, debe ser pensada como un saber más, uno posible de enseñar y aprender, olvidando de alguna manera esa idea de verla como un problema solo de inspiración, un quehacer solo para dotados de nacimiento donde la única palabra parece ser la vocación. Una idea idílica donde no cabe el esfuerzo, el compromiso, la dedicación y el desarrollo de competencias, capacidades y habilidades.

Por consiguiente, la didáctica de la escritura creativa debe tener en cuenta una serie de cuestiones de las que no se escapa la misma práctica educativa, donde se entablan una serie de relaciones complejas como en todo constructo social. Por un lado, su alcance, en este caso la enseñanza y el aprendizaje de la escritura creativa y las relaciones de los participantes con ellos mismos y con el conocimiento, con todo lo que esto significa. Por el otro, la escritura creativa como saber, el cual enfrenta una serie de dificultades por su poca claridad científica y su lucha constante entre concepciones de producción comercial, artística, representación social y cultural; un territorio en disputa entre el lenguaje, el pensamiento, la sociedad y la cultura.

Por esta razón, su enseñanza debe inclinarse hacia el desarrollo del pensamiento como cualquier proceso creativo, no solo estar encaminada al fortalecimiento de las competencias comunicativas y textuales. Debe preocuparse por la subjetividad, promoviendo la imaginación y la autonomía; reconociendo, además, al individuo como parte de un territorio, el cual busca ser representado; y fomentando el placer por la literatura. Así, el enseñante juega un papel fundamental en guiar y contagiar este placer al aprendiz, generando hábitos tanto de lectura como de escritura literaria, permitiendo que este explore y encuentre su propia voz y representación del mundo que lo rodea.

De este modo, una didáctica de la escritura creativa es posible solo permitiendo explorar nuevas formas de enseñar la escritura, no mediante fórmulas de producción que se 
quedan en la idea de réplica, sino mediante la misma creatividad del enseñante, ya que no se puede enseñar a ser creativo sin serlo.

\section{REFERENCIAS}

Alonso, F. (2008). Didáctica de la escritura creativa. Tarbiya, 28, 51-66.

Altamirano Flores, F. (2016). Didáctica de la literatura: ¿cómo se contagia la literatura? La Palabra, (28), 155-171. https://doi.org/10.19053/01218530.4813

Álvarez, M. (2009). Escritura creativa. Aplicación de las técnicas de Gianni Rodari. Educere: Revista Venezolana de Educación, número 44, pp. 83-87. ISSN: 1316-4910. Disponible en: https://www.redalyc.org/articulo.oa?id=356/35614571010

Álvarez, J. \& Cárdenas, J. (enero-junio de 2019). Construir la experiencia de la sed (aproximaciones pedagógicas y políticas a la escritura creativa). La Palabra, (34), 123-132. https://doi.org/10.19053/01218530.n34.2019.9536

Arroyo, R. (2015). La escritura creativa en el aula de educación primaria (tesis maestría). Universidad de Cantabria, Santander, Cantabria, España.

Astolfi, J. P. (1998). Desarrollar un currículo multirreferenciado para hacer frente a la complejidad de los aprendizajes científicos. Enseñanza de las ciencias, 16 (3), 375-385. Disponible en: https://www.raco.cat/index.php/Ensenanza/article/view/21543

Astolfi, J. P. (2001). Conceptos claves en la didáctica de las disciplinas. Sevilla: Diada.

Bordons, G. y Díaz-Plaja, A. (2006). Las aportaciones de la teoría de la literatura a la enseñanza, en Bordons y Díaz-Plaja (coords.) Enseñar literatura en secundaria. Barcelona: Graó.

Briz, E. (2003): Habilidades y competencias lingüísticas. En Didáctica de la Lengua y La Literatura para Primaria, Antonio Mendoza Fillola (aut.), Ezequiel Briz Villanueva (aut.), pp. $175-216$

Camilloni, A.R.W. de (2007). El saber didáctico. Buenos Aires: Paidós.

Canale, M., \& Swain, M. (1981). A Theoretical Framework for Communicative Competence. In Palmer, A., Groot, P. , \& Trosper, G. (Eds.), The construct validation of test of communicative competence, 31-36. 
Cassany, D. (2016). La escritura extensiva. La enseñanza de la expresión escrita en secundaria. Enunciación, 21(1), 91-106. http://dx.doi.org/10.14483/udistrital.jour. enunc.2016.1.a06

Castaño, C.A., \& Fonseca, G. (2008). La didáctica: un campo de saber y prácticas. Bogotá: Universidad Pedagógica Nacional.

Chevallard, Y. (1997). La transposición didáctica. Del Saber Sabio al Saber Enseñado. AIQUE, Buenos Aires.

Comenio, J. A. (2003). Didáctica analítica (Methodi linguarum novissimae fundamentum, ars didáctica). Separata Revista Educación y Pedagogía, 15, 13-87.

Corrales, J. L. (2001). Formación de profesorado en Creación Literaria, Tarbiya: Revista de investigación e innovación educativa, 28, 65-78. Recuperado de: http://dialnet.unirioja. es/servlet/articulo?codigo=237792.

Delmiro Coto, B. (2002). La escritura creativa en las aulas. En torno a los talleres literarios. Barcelona: Graó.

Díaz, P. (2016). Escritura creativa análisis documental sobre las estrategias pedagógicas, propósitos y finalidades formativas en la escuela (tesis de pregrado). Universidad Pedagógica Nacional, Bogotá, Colombia.

Flechsig, K-H. (1985). El Catálogo de Modelos Didácticos de Gotinga (CMDG) - Informe. Educación, 32, 113-118.

Giraldo, C. (2015). La escritura en el aula como instrumento de aprendizaje. Estudio en universidades. Ánfora, 22(38), 39-58. Universidad Autónoma de Manizales. ISSN 0121 6538

Grossman, P.L., Wilson, S.M. y Shulmane, L.S. (2005). Profesores de sustancia: El conocimiento de la materia para la enseñanza. Profesorado. Revista de Currículum y Formación del Profesorado, 9 (2).

Mejía, M. R. (2020). Educación(es), escuela(s) y pedagogía(s) en la cuarta revolución industrial desde Nuestra América. Bogotá, Colombia: Ediciones desde abajo. 
Ong, W. J. (2006). Oralidad y escritura Tecnología de la palabra. Argentina: Fondo de Cultura Económica.

Park, S., Oliver, J. S. (2008). Revisiting the Conceptualization of Pedagogical Content Knowledge (PCK): PCK as a Conceptual Tool to Understand Teachers as Professionals. Research in Science Education, 38 (3), 261-284. https://doi.org/10.1007/s11165-0079049-6

Peña, O.Y., \& Quintero, A.C. (2016). La escritura como práctica situada en el primer ciclo: promoción de procesos cognitivos y metacognitivos. Cuadernos de Lingüística Hispánica, (28), 189-206. http://dx.doi.org/10.19053/0121053X.4915

Rosal, M. (2010). Creación poética y competencia literaria: propuestas didácticas. Álabe, 2. Recuperado de: http://revistaalabe.com/index/alabe/article/view/28

Runge, A. K. (2013). Didáctica: una introducción panorámica y comparada. Itinerario Educativo, 62, 201-240.

Shulman, L.S. (1987). Knowledge and teaching: Foundations of the new reform. Harvard Educational Review, 57 (1), 1-22. Edic. cast: Conocimiento y enseñanza: fundamentos de las nueva reforma. Profesorado. Revista de Currículum y Formación del Profesorado, 9 (2), 2005.

Sternberg, R. J. (1997). Successful intelligence. New York: Plume.

Tabarovsky, D. (2004) Literatura de Izquierda, Rosario: Beatriz Viterbo.

Timbal-Duclaux, L. (1993). Escritura creativa (Vol. 3). Edaf.

Vasco, C. (1990). Reflexiones sobre pedagogía y didáctica. Serie Pedagogía y Currículo, Bogotá: Ministerio de Educación Nacional-Dirección General de Capacitación.

Vasco, C., Martínez, A., \& Vasco, E. (2008). Educación, pedagogía y didáctica: una perspectiva epistemológica. En G. Hoyos (Ed.), Filosofía de la Educación. (pp. 99-127). Madrid, España: Editorial Trotta. 
Velásquez, B., Remolina, N., \& Calle, M. (2010). La creatividad como práctica para el desarrollo del cerebro total. Tabula Rasa, (13), 321-338. Recuperado de: https://revistas. unicolmayor.edu.co/index.php/tabularasa/article/view/1435

Zambrano, A. (2005). La didáctica "lugar en las ciencias de la educación". En: Los hilos de la palabra: pedagogía y didáctica (pp. 167-204). Bogotá: Editorial magisterio.

Zambrano, A. (2006). Las ciencias de la educación y didáctica: hermenéutica de una relación culturalmente específica. Revista Venezolana de Educación Educere, 10(35), 593599. 